\title{
Motilidad, flujo intestinal y patrón de ácidos biliares en niños con síndrome diarreico*
}

Algunos de los mecanismos patogénicos que se han postulado para explicar ciertos tipos de Síndrome Darreico Crónico (SDC) pueden verse en la figura 1. Cualquier noxa primaria como las señaladas: infecciones bacterianas o virales ${ }^{1}$, cirugía abdominal con manipulación de asas intestinales ${ }^{1}$, drogas como derivados atropínicos o colestiramina ${ }^{2-3}$ y otras como hipercontaminación ambiental o hipokalemia ${ }^{1}$, pueden provocar uno o más de los siguientes fenómenos: hipomotilidad, sobrecrecimiento bacteriano y un patrón anormal de ácidos biliares. Cualquiera de estos fenómenos puede desencadenar o potenciar el otro, dando lugar a malabsorción con o sin daño del epitelio intestinal, resultando finalmente en un síndrome diarreico crónico.

En estudios de perfusión intestinal en ninos con síndrome diarreico, Torres Pinedo demostró la existencia de un retardo en el tiempo

\footnotetext{
*Trabajo parcialmente financiado a través del fondo de ayuda para adiestramiento Instituto Nacional de Salud AMO5721-02 E.U.A. y Universidad del Zulia, Maracaibo Venezucla.

** Sección de Nutrición y Gastroenterología, Departamento de Pediatría, Baylor College of Medicine, Houston, Texas, y Unidad de Estudios Clinicos. Hospital Roosevelt. Guatemala, Guatemala.

***Departamento de Pediatría, Hospital Ruberto del Rio.
}

Jesús L. Cupello**, Jorge T. Rodríguez**, Felipe N. Flores**, Tzu Lee Huan**, Humberto Soriano *** y Buford L. Nichols**

de tránsito de intestino delgado ${ }^{4}$. No hemos encontrado otras referencias en la literatura en las que se haya medido el tiempo de tránsito de intestino delgado en niños con síndrome diarreico agudo. Los estudios de tránsito intestinal total (boca-ano) $)^{5}$ en niños con síndrome diarreico agudo, el tránsito intestinal fue encontrado muy variable, pudiendo estar normal, acelerado o retardado. Este mismo hallazgo ha sido reportado por Viteri y col., en niños desnutridos con o sin diarrea ${ }^{6}$. Por fenómenos aún no del todo bien conocidos, el sobrecrecimiento bacteriano en el intestino delgado superior, puede interferir con la digestión y absorción de nutrientes o ser causa de hipersecreción de agua y de electrólitos ${ }^{7-8 \cdot \theta}$, desencadenando diarrea y esteatorrea. Uno de los mecanismos por los cuales el sobrecrecimiento bacteriano puede afectar la función intestinal, es a través de la deconjugación precoz y del metabolismo anormal de ácidos biliares provocado por la flora bacteriana inhabitual en el intestino delgado superior. Esto conduce al aumento de ácidos biliares libres en el lumen intestinal y a la producción de ácidos biliares secundarios.

La deconjugación de ácidos biliares por bacterias, aumenta la proporción de ácidos biliares secundarios ${ }^{799}$. Algunos de ellos como el de- 


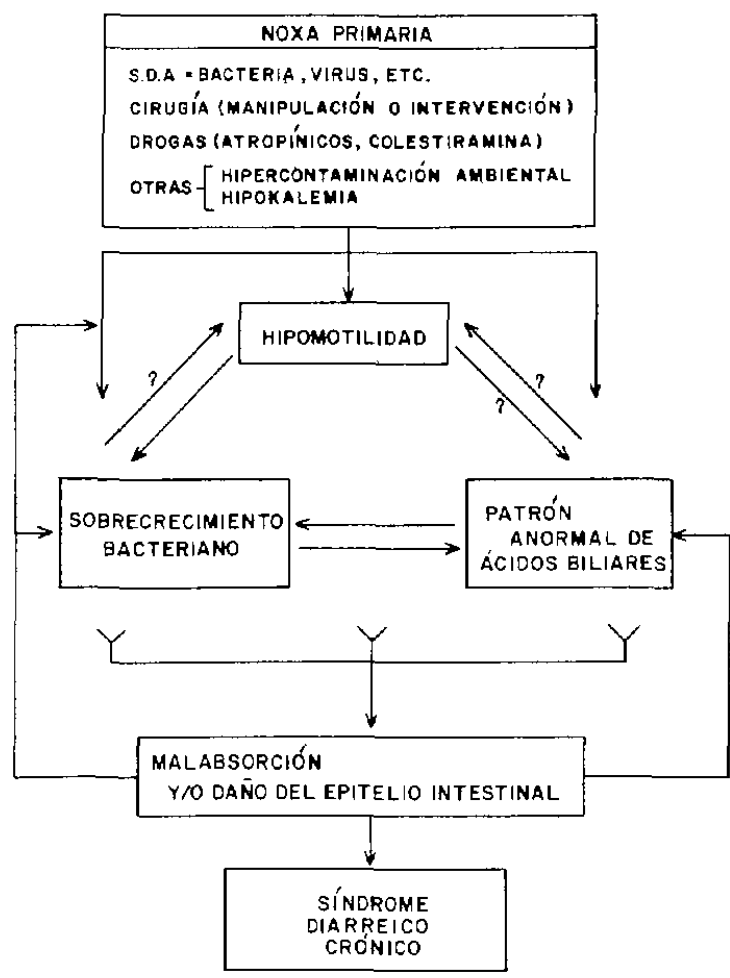

oxicólico y el litocólico ha sido demostrado que tienen efectos tóxicos sobre la mucosa y su función ${ }^{6-10}$, y tambièn han sido hallados inhibidores de la absorción de agua y sales ${ }^{7}$.

El presente estudio se realizó con el propósito de investigar los mecanismos patogénicos anteriormente mencionados en el síndrome diarreico agudo del niño, mediante la medición del tiempo de tránsito en 1 metro de intestino, el patrón de ácidos biliares en el contenido duodenal y el flujo de agua a nivel del íleon.

\section{Material y Métodos}

Catorce niños guatemaltecos fueron estudiados durante el período diciembre 74 - febrero 75 en la Unidad de Estudios Clínicos del Hospital Roosevelt, de Guatemala. Sus edades estuvieron comprendidas entre 6 meses y 6 años, 9 del sexo masculino y 5 del sexo femenino. Todos presentaron diarrea como síntoma principal, sólo 2 presentaron diarrea con moco y sangre. El antecedente de vómitos se encontró en 12 de ellos y 9 presentaron fiebre a su ing reso (tabla 1). Estos pacientes fueron seleccionados de la sala de observación donde recibían líquidos endovenosos para corregir su grado de deshidratación. Se consiguió permiso escrito de los padres para proceder a practicar intubación duodenal con una sonda de doble lumen. El protocolo de estudios fue aprobado por el comité de investigaciones humanas del hospital.

De los 14 niños, 9 de ellos resultaron con coprocultivo negativo para gérmenes enteropatógenos, 4 de ellos con crecimiento de Escherichia Coli serotipo positivo (ECP) y 1 con crecimiento de Shigella. Con el objeto de establecer comparación de los parámetros estudiados se utilizaron los datos de 7 niños con síndrome diarreico crónico no específico y 4 niños sin patología digestiva previamente estudiados por nuestra sección en el mismo hospital. Todos los pacientes se intubaron utilizando una sonda de silástico de doble lumen de $2 \mathrm{~mm}$ de diámetro externo y $1,5 \mathrm{~mm}$ de diámetro interno. Los orificios proximales se colocaron en el duodeno y los distales $100 \mathrm{~cm}$ más abajo (figura 2). Después de colectar muestras duodenales para medir ácidos biliares, se administró por vía intragástrica una solución $(25 \mathrm{ml})$ de Poli-etilen-glicol al $4 \%$ como marcador no absorbible. Durante 4 y media horas se colectaron muestras procedentes de ambos sitios. La aparición del Poli-etilen-glicol (PEG) en el duodeno y $100 \mathrm{~cm}$ más abajo permitió calcular el tiempo de tránsito. El flujo ileal fue calculado de acuerdo al método de Hamil$\operatorname{ton}^{11}$.

$\frac{\text { Flujo ileal }}{\mathrm{ml} / \mathrm{mm}}=\frac{\text { PEG oral total en mgs }}{(\text { PEG } \times) \text { Ileon } \mathrm{mg} / \mathrm{ml} \times \text { Tiempo minutos }}$

o sea, PEG oral total en miligramos dividido por promedio de $\mathrm{PEG}$ en íleon en miligramos por $\mathrm{ml}$, multiplicado por tiempo en aparecer y desa. parecer en minutos. La concentración de PLG fue calculada de acuerdo al método turbidimétrico de $\mathrm{Hyden}^{12}$. Los ácidos biliares se determinaron por la técnica de cromatografía de gas líquido de acuerdo a la técnica de Kaskis y Youseff $^{13}$.

\section{Resultados}

En los casos de síndrome diarreico crónico inespecífico se encontró retardo en el tiempo de 
Tabla 1

\begin{tabular}{|c|c|c|c|c|c|c|c|}
\hline Pacientes & Edad & Peso & Sexo & $\begin{array}{c}\text { Estado } \\
\text { nutricional }\end{array}$ & $\begin{array}{c}\text { Promedio } \\
\text { evacuaciones }\end{array}$ & Fiebre & Vómitos \\
\hline 1) $\mathrm{CC}$ & 20 meses & $8.100 \mathrm{grs}$ & Mas. & Desnutrido II Grado & 10 al dia & Sí & Sí \\
\hline 2) $\mathrm{EG}$ & 9 meses & $7.100 \mathrm{grs}$ & Mas. & Desnutrido I Grado & 20 al día (sangre) & Sí & Si \\
\hline 3) $\mathrm{EC}$ & 7 meses & $5.450 \mathrm{grs}$ & Mas. & Eut rófico & 10 al día (sangre) & Sí & Sí \\
\hline 4) $\mathrm{MC}$ & 13 meses & $6.800 \mathrm{grs}$ & Mas. & Desnutrido II Grado & 8 al día & Si & No \\
\hline 5) $\mathrm{MV}$ & 6 años & $10.600 \mathrm{grs}$ & Fem. & Desnutrida III Grado & 8 al día & Sí & Sí \\
\hline 6) JC & 21 meses & $6.100 \mathrm{grs}$ & Mas. & Desnutrido II Grado & 10 al día & Sí & Sí \\
\hline 7) $\mathrm{RG}$ & 7 meses & $6.600 \mathrm{grs}$ & Fem. & Eutrófica & 6 al día & Sí & Sí \\
\hline 8) $\mathrm{CG}$ & 11 meses & $6.700 \mathrm{grs}$ & Mas. & Desnutrido I Grado & 4 al día & No & No \\
\hline 9) $\mathrm{SM}$ & 14 meses & $6.500 \mathrm{grs}$ & Fem. & Desnutrida II Grado & 6 al día & Sí & Sí \\
\hline 10) $\mathrm{AH}$ & 16 meses & $7.100 \mathrm{grs}$ & Mas. & Desnutrido II Grado & 8 al día & Si & No \\
\hline 11) BG & 11 meses & $6.100 \mathrm{grs}$ & Fem. & Desnutrida I Grado & 5 al dia & Sí & Sí \\
\hline 12) MV & 12 meses & $6.800 \mathrm{grs}$ & Fern. & Desnutrida II Grado & 8 al día & No & Sí \\
\hline 13) NR & 16 meses & $7.300 \mathrm{grs}$ & Mas. & Desnutrido II Grado & 6 al día & No & Sí \\
\hline 14) $\mathrm{HP}$ & 16 meses & $7.200 \mathrm{grs}$ & Mas. & Desnutrido II Grado & 4 al día & No & Sí \\
\hline
\end{tabular}

Figura 2

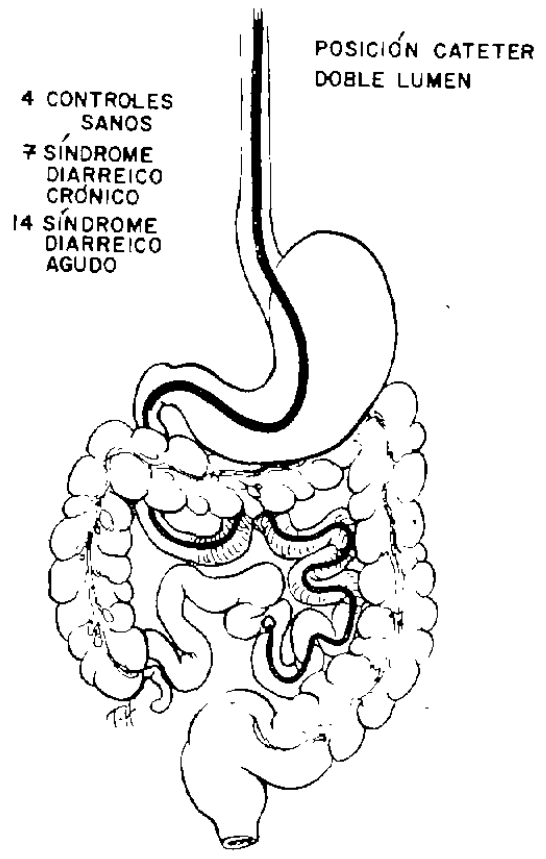

tránsito intestinal de más de 90 minutos por metro si se comparaban con 60 minutos que se encontraron en los controles sanos. En los casos de síndrome diarreico agudo, se encontraron valores diferentes de acuerdo con el tipo de patógenos encontrados (figura 3) 90 minutos de promedio en los casos de síndrome diarreico agudo inespecífico, 80 minutos de promedio en los casos de Escherichia coli serotipo positivo y acelerado en el caso de Shigella con 30 minutos por metro. En la figura 4 se puede ver la aparición y desaparición del PEG mucho más rápido en el niño con shigellosis, comparada con los niños con ECP y el grupo de niños con síndrome diarreico agudo inespecífico.

En los pacientes con diarrea crónica y en todos los pacientes con sindrome diarreico agudo el patrón de ácidos biliares fue anormal comparado con el grupo control (tabla 2). El porcentaje de ácidos biliares libres fue significativamente mayor; el $\Delta^{5} 3^{B}$ estuvo también aumentado 

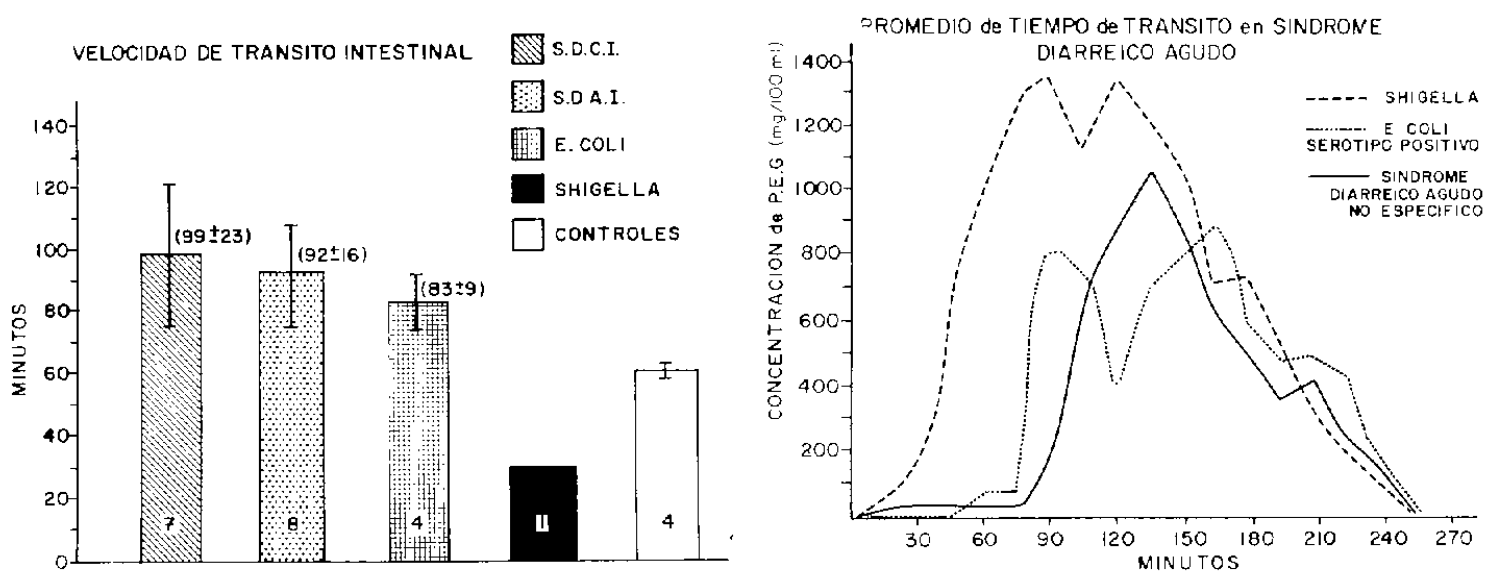

Tabla 2

PATRON DE ACIDOS BILIARES EN DUOIDENO DE NIÑOS CON SINIDROME DIARREICO AGUDO

\begin{tabular}{|c|c|c|c|c|}
\hline$\% A B$ & $\operatorname{SDAI}(N=7)$ & $\operatorname{ECEP}(n=1)$ & Shigella & Controtes i $n=4$ ) \\
\hline I.C & $23,5 \pm 4,9$ & $17,6 \pm \quad 3,1$ & $59,6 \%$ & $10,3 \pm \quad 3,0$ \\
\hline$\Delta^{5}, 3 \mathrm{~B}$ & $4,0 \pm 1,1+$ & $4,0 \pm \quad 0,7 i$ & 1.6 & 0 \\
\hline 1501000 & $15,9 \pm 2,4$ & $11,3 \pm \quad 4,6$ & 9 & $15,0 \pm \quad 6,8$ \\
\hline DOC & $0, \overline{5} \pm 0.2$, & $1,5 \pm 1,1+$ & 0 & $20,7 \pm \quad 6,6$ \\
\hline CDC: & $14,8 \pm 2.2$ & $19,5 \pm 3,1$ & 4,7 & $17,7 \pm \quad 3,2$ \\
\hline URSOIDOC & $1,1 \pm 0,1$ & $3,5 \pm 2,7$ & 0 & $6,9 \pm \quad 3,7$ \\
\hline C: & $38,7 \pm 5,2$ & $40,7 \pm \quad 2,9$ & 16,6 & $24,6 \pm 2.6$ \\
\hline $\begin{array}{l}\text { lotal } \mathrm{AB} \\
(\mu \mathrm{g} / \mathrm{ml})\end{array}$ & $340,0 \pm 66,0$ & $724,0 \pm 254,0$ & 64,3 & $457,0 \pm 234,0$ \\
\hline$\%$ Libre & $25,7 \pm 2,3^{*}$ & $26,1 \pm 8,1+$ & 72,3 & $3.2 \pm 1,1$ \\
\hline $\mathrm{G} / \mathrm{T}$ & $15,0 \pm 7,7$ & $5,0 \pm 1,0+$ & 0 & $2,5 \pm 0,3$ \\
\hline c/Col: & $3,0=0,5$ & $2,3 \pm 0,4$ & 3,57 & $\mathrm{i}, 4 \pm \quad 0,3$ \\
\hline
\end{tabular}

Diferencia significativa comparada con los cont roles: $\mathrm{p}<0,05 ; \therefore \mathrm{p}<0.01 ;$ i $\mathrm{p}<0,005 ;{ }^{*} \mathrm{p}<0,001$

to mismo que los ácidos biliares conjugados con glicina. Se encontró un deoxicólico disminuido en los casos de diarrea aguda y crónica.

El flujo ileal se encontró variable de acuerrio al tipo de patógeno: $0,6 \mathrm{ml} / \mathrm{min}$ en los casos de Shigella, $1,07 \mathrm{ml} / \mathrm{m}$ in en los casos de Escherichia coli, y $1,3 \mathrm{ml} / \mathrm{min}$ en los casos de síndrome dia rreico agudo inespecífico (tabla 3 ).

Discusión

El tracto gastrointestinal es un complejo sistema ecológico en donde muchos factores interaccionan entre sí para mantener la normalidad de los procesos de absorción y secreción. En algu- nos tipos de síndrome diarreico pueden existir cambios importantes en la calidad y cantidad de microflora presente en el intestino delgado condicionando malabsorción y/o hipersecreción por mecanismos aúr no del todo bien precisa$\operatorname{dos}^{6-7-8}$.

Los movimientos del intestino delgado superior probablemente juegan un papel muy importante en la acción limpiadora impidiendo el sobrecrecimiento bacteriano. Entidades clínicas que reducen la motilidad intestinal como el sindrome de asa ciega ha sido hallada con sobrecrecimiento bacteriano exagerado ${ }^{3}$. Otras entidades como el Esprue Tropical y la Desnu- 
Tabla 3

TRANSITO INTESTINAL ${ }^{a}$ Y FLUJO ILEAL ${ }^{\circ}$ EN NIÑOS CON SINIDROME I)IARREICO

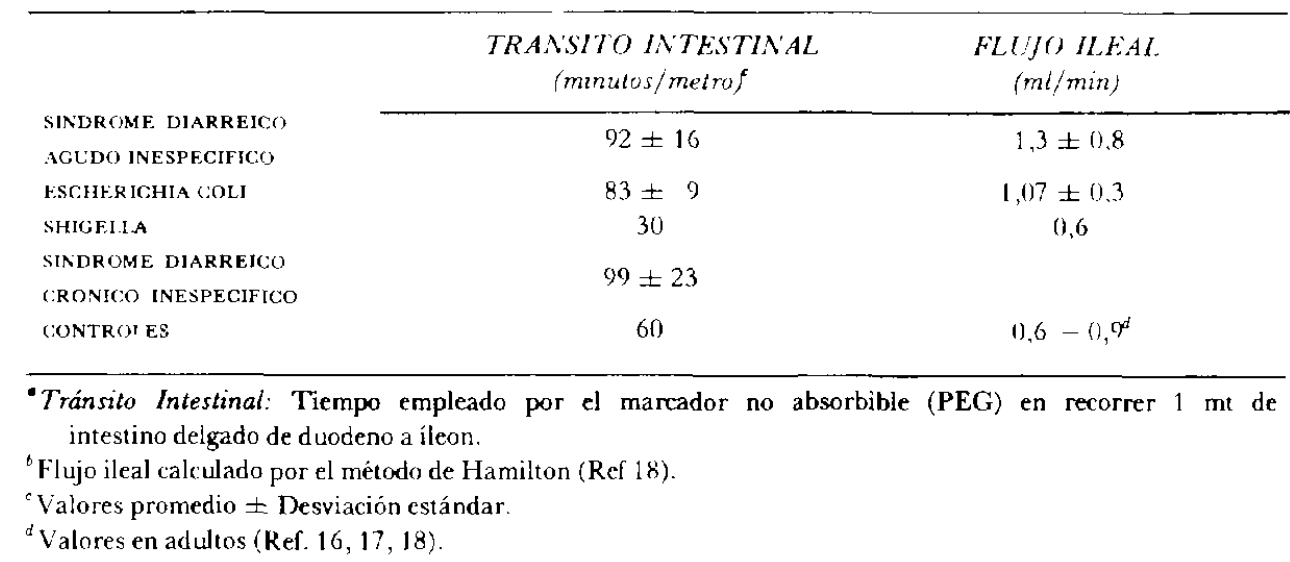

nutrición Proteico Calórica, también han sido halladas con sobrecrecimiento bacteriano en el intestino delgado ${ }^{6}$. En nuestro grupo de niños con sindrome diarreico agudo así como también los crónicos que viven en áreas de hipercontaminación ambiental, el sobrecrecimiento bacteriano del intestino delgado podría tener un importante papel patogénico a través de la deconjugación y formación de ácidos biliares libres en forma prematura ${ }^{\mathbf{B} 9}$. Esto podría por una parte alterar la fase intraluminal de la digestión grasa y, por otra parte, provocar por sí nismo alteración morfofuncional del epitelio intestinal $1^{14-15}$.

La hipomotilidad encontrada en nuestros pacientes (con excepción del caso de shigella) vendría a favorecer el sobrecrecimiento bacteriano que ya de por si puede existir en nuestros pacientes por vivir en ambientes hipercontaminados. $\Lambda$ demás el $85 \%$ de nuestros pacientes estudiados con síndrome diarreico agudo fueron desnutridos, que de por sí tienen hipomotili$\operatorname{dad}^{6}$. En el caso de Shigella, aun cuando su acción patogénica principal es a nivel del colon invadiendo la mucosa y provocando colitis infecciosa, el hallazgo de aceleración del tiempo de tránsito en el intestino delgado puede jugar un papel patogénico importante en cuanto a malabsorción de nutrientes.

Los llujos de agua a nivel ileal se encontraron variables: $0,6 \mathrm{ml} / \mathrm{min}$ en el caso de Shigella que fue estudiada 4 días después de su ingreso cuando su acción patogénica era netamente co- lónica. Los casos de Escherichia coli 1,07 $\mathrm{ml} / \mathrm{min}$ y los casos de síndrome diarreico agudo inespecífico $1,3 \mathrm{ml} / \mathrm{min}$, representan valores de flujo ileal aumentados si se comparan con los valores encontrados en adultos sanos por Devroede $0,6 \mathrm{ml} / \mathrm{min}^{16}$, Whalen $0,67 \mathrm{ml} / \mathrm{min}^{17}$ y Giler $0,9 \mathrm{ml} / \mathrm{min}^{18}$.

No hemos encontrado en la literatura ninguna referencia de flujo ileal en niños sanos o con síndrome diarreico. Estos resultados señalan que en niños con síndrome diarreico agudo donde no se aislaron patógenos y en los niños donde se aisló Escherichia coli llamada enteropatógena, hubo un estado de hipersecreción intestinal. Este hecho concuerda con la hipótesis de una acción enterotóxica sobre la mucosa del intestino postulada por otros autores ${ }^{8}$. El caso de Shigella estudiado revela que en el período de estado de la shigellosis la hipersecreción del intestino delgado no es un factor importante en la producción del síndrome diarreico.

Los ácidos biliares primarios cólico y quenodeoxicólico son sintetizados por el hígado $y$ son secretados conjugados con glicina y taurina. Los ácidos biliares secundarios como el doxicólico y el litocólico son producidos en el lumen intestinal por enzimas producidas por bacterias, las deshidroxilasas al actuar sobre los ácidos biliares primarios. I.os ácidos biliares libres y secundarios no toman parte en la formación de micelas, así que no intervienen en la absorción de grasa. Algunos de éstos han sido hallados in- 
hibidores del transporte de glucosa y aminoácidos, así como también productores de daño del epitelio intestinal estimulando secreción de $\operatorname{agua}^{10}$.

En nuestros pacientes se encontró un aumento significativo en el porcentaje de ácidos biliares libres, lo que podría estar dado por sobrecrecimiento de la flora bacteriana a este nivel. Junto con esto el aumento de los ácidos biliares secundarios o terciarios $\left(\triangle^{5} 3^{B}\right)$ y de los conjugados con glicina apoyan la hipótesis de una mayor actividad metabólica de la flora microbiana a nivel del intestino delgado. Por último, el hallazgo de una disminución en el porcentaje de ácido deoxicólico podría estar condicionado por una menor absorción (con mayor pérdida fecal) o menor capacidad de síntesis hepática de este ácido.

\section{Conclusiones:}

1. El síndrome diarreico del niño puede presentarse con tiempo de tránsito intestinal acelerado o retardado según su etiología.

2. En el síndrome diarreico del niño, el pat rón de ácidos biliares en el intestino delgado es anormal, lo que podría traducir un sobrecrecimiento bacteriano a ese nivel y desempeñar un papel patogénico en esta enfermedad.

3. El flujo de agua a nivel del íleon está aumentado en todos los casos de diarrea con excepción del paciente con Shigella.

\section{AGRADECIMIENTOS}

Los autores agradecen a la señora Jo Ann Christian por su excelente trabajo de secretaria.; al Dr. Humberto coriano por la asistencia editorial.

\section{SUMMARY}

Fourtecn children between the ages of six months and six years with acute diarrheal syndrome were investigated for Small Bowell Transit Time (SB'TI). The results were rompared with another study made by our section in which elcven children were studied; seven with chronic nonspecific diarrhea and four controls. All subjects had a double lumen catheter positioned in the small intestine with pory located 1 meter apart. A bolus of $4 \%$ PEG was delivered into the stomach and the rate of appearance was monitored at the two collecting ports. Four normal children had a small bowel transit time of $60 \mathrm{~min} / \mathrm{m}$; one subject with shigella revealed a small bowed transit time of $30 \mathrm{~min} / \mathrm{m}$. Nine children with acute non-specific diarthea had a mean of 90 . Seven with chronic non-specific diarthea had a mean of $99 \mathrm{~min} / \mathrm{m}$. Four patients with serotype positive l:. coli had a mean of 83 . In each case where SB'IT was delayed there was also an increase in luminal free bile acids. Ileal water flux was increased compared with that seen in normal adults except for shigella.

\section{CONCLUSION}

Diarrheal syndrome in children may present wit SBTT accelerated or delayed according to the etiology of each. Children with diarrheal syndrome showed an abnormal bile acid pattern in the duodenum wich may represent a bacterial overgrowth and introduce a pathogenic role in the disease. lleal flux was increased in acute non-specific diarrhea and E. coli.

\section{REFERENCIAS}

Conel, A.M. Medical Clinics of North America, 58 (6): 1201-15, November. 1974.

2 West, R.J. and Lloyd, J.K. Gut, 16:93-98, 1975.

3 Hofmann, A.F. and Rainer, P.J. Gastroenterology. 62(5):918-34, 1972 .

- Torres-Pinedo, R.; Ruvera, $C$. and Rodríguez, $H$. Annals New York Academy of siciences, 176:284-98. January 7, 1971.

- Daffau, G.; Macaya, J. y Soriano, H. Pediatría (Santiago de Chile). 15:445-51, 1972.

- Viter, F.E. and Schneider, R.E. Medical Clinics of North America, 58(6):1487-1504, November, 1974.

7 Low Beer, T.S. and Read, A.E. Gut, 2:1021-36.

- Fagundes, N.L. Acta Gastroenterológica Lat. América, 5:91-3, 1973.

- Rodríguez, J.T.; Ordóñez, J.V.; Huang, T.L.; Alvarato $\mathrm{J}$. and Nichols, B.I. Intestinal Microflora, Bile Acid Patterns and Small Bowel Transit Time in Guatemalan Children with Chronic Non-specific Diarrhea and Healthy Controls. Ped. Wr (Buenos Aires, Argentina, 1974) (Gastroenterología).

10 Rusell, R.l. Scot Medical Journal, 18:146-51, Januars, 1973

11 Hamelton, W'F. (Section Editor) Circulation. Vol. $\mathrm{I}$, in Handbook of Physiolog, Section II Washington American Physiology Society 1962, pp. 573-80.

12 Iyden, S.A. Ann. Agr. Coll. Sweden, 22:139, 1955.

13 Youseff, I.H.; Kaskis, (A. and Fisher. Can. J. Bionhem.. $50: 4(12-8,1972$.

14 Campbell, C:B.; Coumen, I.E. and Haper, J. Australian N.A.j. Med., 3:339-48, 1973.

15 (irace), M. Gut, 12:403-10, 1971.

16 Devroede $G$. and Phullips, S.F. Ciastronterology, Vol. $56(1): 92-100.1969$.

17 Whalen G.E.; Harris, J.A.; Geenen, J.E. and Soergel, K.H. Gastroenterology, Vol. 51(6): 975-84. 1966.

18 Giller, J. and Phillips, S.F. Gastroenterology, June 1970. page 95. 